\title{
Women's decision making about hormone replacement therapy balanced being in control with fear, uncertainty, and dislike of medications
}

Griffiths F. Women's control and choice regarding HRT. Soc Sci Med 1999 Aug;49:469-81. QUESTION: What is the process women go through when deciding whether to begin
hormone replacement therapy (HRT) (either oestrogen and progesterone or oestrogen
alone)?

Design
Qualitative study using individual interviews and focus
groups.

Setting

Community study in Stockton-on-Tees, UK.

\section{Participants}

1649 women aged 20-69 years who were part of 8 general practices were sent questionnaires on their attitudes and knowledge about health and HRT. 1225 women completed the questionnaire and from these women, a stratified random sample was drawn to identify 17 women who were interviewed and 25 women who participated in 1 of 5 focus groups. All women who were interviewed or in the focus groups were white European.

\section{Methods}

Interviews focused on the women's personal stories and included discussion of general attitudes to health, development of attitudes toward HRT, and what had influenced the HRT decision. Focus groups were held to explore the complex motivations or attitudes around the use of HRT. Information from both processes was analysed using the immersion/crystallisation analysis method. Themes and factors that influenced attitudes to medication in general, and HRT in particular, were identified.

\section{Main findings}

Data from the interviews found that 8 women felt they disliked medication in general and they were not taking HRT; 7 women were taking HRT. Regardless of HRT status, women held a wide range of attitudes, both positive and negative, toward HRT. Themes common to all of the women were fear of illness, disability, or death; factors that prompted or increased the fear; how fear was controlled; and what reduced the fear. Fear was linked to attitudes and their own experiences with ill health as well as those of friends and family. Sources of information about HRT were the media, work and social contacts, background and training, and contact with their physicians.

In the focus groups, 4 women were taking HRT and 1 woman was a former user. The theme of control (choice about whether to take any medication including HRT) was identified. Control was somewhat reduced by health factors (eg, family history of osteoporosis or need for a hysterectomy), social attitudes (eg, menopause being the end of active life), and fear and uncertainty. Factors relating to fear and uncertainty were similar in women who were interviewed and those who were in the focus groups. Increased control was attained by finding information, or changing work patterns or other environmental factors.

\section{Conclusions}

In deciding whether to begin hormone replacement therapy, women considered and balanced feeling in control, fear and uncertainty related to ill health and personal experiences, and dislike of medications. Media and social contacts were important sources of information.

\section{COMMENTARY}

The research completed by Griffiths provides additional support for the complexity of women's decision making processes when considering the use of HRT. The findings are consistent with previous work on decision making regarding HRT. ${ }^{1-2}$ Additionally, Griffiths found that the findings do not completely support the medicalisation of menopause. On the contrary, although the women sought the expert opinion of their physicians, they were more likely to make decisions based on the context within which they lived. This finding stresses the importance of listening to and knowing women as individuals who bring their unique context to every health related decision making process.

Griffith cautions the reader with regard to the interpretation of the findings and the application to practice. Generally, the findings are limited by self selection, a sampling technique that focused on women with strong opinions about HRT, and participant awareness that the individual conducting the interviews and focus groups was a physician. Despite these limitations, the findings do suggest that primary care providers need to attend to women's concerns when considering alternatives for treatment of menopausal symptoms. ${ }^{3}$ Knowing that the media, experience, social contacts, and physicians influenced the women's decisions can guide interactions between healthcare providers and women. Focused questions will encourage women to discuss what they have learned from these resources, help to clarify misconceptions, and identify the need for additional information. In turn, healthcare providers can attend to the need women have to feel in control of their decisions by supporting their need to make these decisions "by themselves".

This study raises questions about the influence of attitudes towards medication in general and how these attitudes might influence the women's decision making processes and their ability to follow treatment regimens that may be needed for successful outcomes. Finally, the influence of personal control on the ability of women to experience comfort in their decisions warrants further investigation.

Karen Moore Schaefer, RN, DNSc Assistant Professor for Nursing Temple University, College of Allied Health Philadelphia, Pennsylvania, USA

Source of funding: Economic and Social For correspondence: Dr F Griffiths, Primary Care Unit, School of Postgraduate Medicine University of Warwick, Coventry CV4 7AL, UK. 